Jain, Ajay

[18]

\section{An analytical framew ork and data system for analyzing megavariate biological data in cancer}

\author{
Ajay Jain, Chris Kingsley \& Adam Olshen \\ University of California San Francisco Cancer Center, San Francisco, \\ California, USA
}

Techniques for in vitro genomic and proteomic analysis are generating large amounts of quantitative biological data, with high-density DNA microarrays capable of producing 30,000 measurements from a single sample of RNA. Such data offer fertile ground for systematic computational analyses to identify new cancer targets or potential therapeutic agents. For such techniques to be most useful, computational methods must generate conclusions that are quantitatively supportable in a rigorous statistical sense, not just provide a means of visualization. False patterns may arise when the ratio between the number of measurements and the number of experimental samples is very high. We present a general method for rigorously identifying correlations between variation in high-bandwidth biological measurements and outcomes or phenotypes. We apply the technique to DNA copy number data and expression data from multiple malignancy types. We demonstrate identification of specific abnormalities linked with poor outcomes, as well as automatic classification of tumor samples by phenotype using an augmentation of the method. The techniques have been implemented in the context of an integrated Web-based data system designed to facilitate interaction between biologists and computational researchers.

Jazaeri, Amir

\section{Gene expression profiles of low - and high-grade papillary serous ovarian carcinomas using CDNA microarray analysis}

Amir Jazaeri ${ }^{1,2}$, Karen Lu², Christos Sotiriou ${ }^{1}$, Nawal Alkharouf ${ }^{1}$, David Gershenson ${ }^{2} \&$ Edison Liu ${ }^{1}$

${ }^{1}$ National Cancer Institute, National Institutes of Health, Bethesda, Maryland, USA ${ }^{2}$ M.D. Anderson Cancer Center, Houston, Texas, USA

We have investigated the use of complementary DNA microarrays in revealing patterns of gene expression in low- and high-grade serous papillary ovarian carcinomas. We used frozen tumor samples from eight ovarian and three peritoneal serous papillary adenocarcinomas. We extracted total RNA from the samples using Trizol and used pooled RNA from two immortalized ovarian surface epithelial cell lines as a reference. Tumor and reference RNAs were amplified using a modified Eberwine method. Cy3 (reference) or Cy5 (samples) was used to label $4 \mu \mathrm{g}$ of amplified RNA, and a 6,500-cDNA microarray chip was used to compare each sample to the reference. We observed a high degree of similarity in the gene expression profiles of low- and high-grade tumors. Forty-five outliers (fourfold up- or downregulated compared with the reference in all 11 samples) were identified. The overexpressed outliers included those encoding CTBP-1, Jun-D, MADCAM-1, and Wilms tumor zinc-finger protein. Genes showing reduced expression included those for IL-8, metallothionein 1X, CDC25B, and uteroglobin. We explored possible differences between the low- and high-grade samples by performing Wilcoxon analysis on each gene in the two groups. This step yielded 27 genes that were differentially expressed $(P<0.03)$ among all low- and high-grade cancers and were sufficient for separating them on multidimentional scaling. Many of the overexpressed genes may be used as tumor markers or serve as therapeutic age
Jeffs, Aaron

[20]

\section{Microarray analysis of colorectal cancer cell lines}

\author{
Aaron Jeffs ${ }^{2}$, Sharon Pattison ${ }^{1}$, Junjie $\mathrm{Xu}^{1} \&$ Anthony Reeve ${ }^{1}$ \\ ${ }^{1}$ Cancer Genetics Laboratory, Department of Biochemistry, University of Otago, \\ Dunedin, New Zealand²Otago Genomics Facility, Department of Biochemistry, \\ University of Otago, Dunedin, New Zealand
}

The incidence of colorectal cancer (CRC) in New Zealand non-Maori women is recognized as the highest in the world. Further, the highest global rates of CRC occur in New Zealand and Australian populations. We are profiling the gene expression of CRC cell lines using CDNA microarrays with the aim of providing baseline data for a clinical investigation into the genetic events involved in metastasis of this disease. Presently, the genetic mechanisms behind metastasis of CRC are poorly understood. The development of metastasis significantly reduces fiveyear survival of CRC patients from at least $70 \%$ with no metastatic involvement, to $35-65 \%$ with lymph node involvement, and $5 \%$ with distant metastasis. We have generated cell lines from patient tumour samples with varying Dukes classification of disease pathology. Of particular interest are two cell lines from the same individual obtained one year apart that are derived from non-metastatic CRC at the Dukes B stage, and metastatic CRC at the Dukes C stage. Gene expression data comparing the metastatic and non-metastatic cell lines will be presented. Greater understanding of the molecular processes involved in the initiation and progression of CRC may provide new clinical options for CRC screening and treatment, and for prediction and prevention of metastatic events in CRC.

Joseph, Pius

[21]

\section{Gene expression in BALB/c-3T3 cells transformed with beryllium}

\section{Pius J oseph \& Tong-man Ong}

Toxicology and Molecular Biology Branch, National Institute for Occupational Safety and Health, Centers for Disease Control, Morgantown, West Virginia 26505, USA

We investigated differential gene expression as a possible molecular mechanism responsible for cell transformation induced by beryllium. Using the Atlas Mouse 1.2 Microarray (Clontech), we studied expression of genes involved in cancer, stress response and DNA synthesis, repair and recombination in BALB/c-3T3 cells transformed with beryllium sulfate. Genes whose expression was at least twofold different in the transformed cells compared with nontransformed cells were as follows: cancer-related genes (9): ets-related transcription factor gene activated by ras, colony-stimulating factor gene, $A-m y b$, sky, cot, c-fos, c-jun, c-myc and R-ras proto-oncogenes; stress response genes (5): HSP84, HSP86, heme oxygenase 2 gene, etoposide-induced p53-responsive gene and oxidative stress-induced gene; and DNA synthesis, repair and recombination genes (11): genes for MCM4 DNA replication licensing factor, MCM5 DNA replication licensing factor, PMS2 DNA mismatch repair, DNA excision repair, MSH2 DNA mismatch repair, Rad23 UV excision repair, Lupus Ku autoantigen, DNA ligase 1, Rad51 and Rad52. We have also found that the beryllium-induced transcriptional activation of c-fos and c-jun is dependent on pathways of protein kinase $\mathrm{C}$ and MAP kinase and independent of reactive oxygen species. These results indicate that alterations in the expression of genes related to cancer, stress response and DNA damage repair may be responsible for the cell transformation induced by beryllium. 